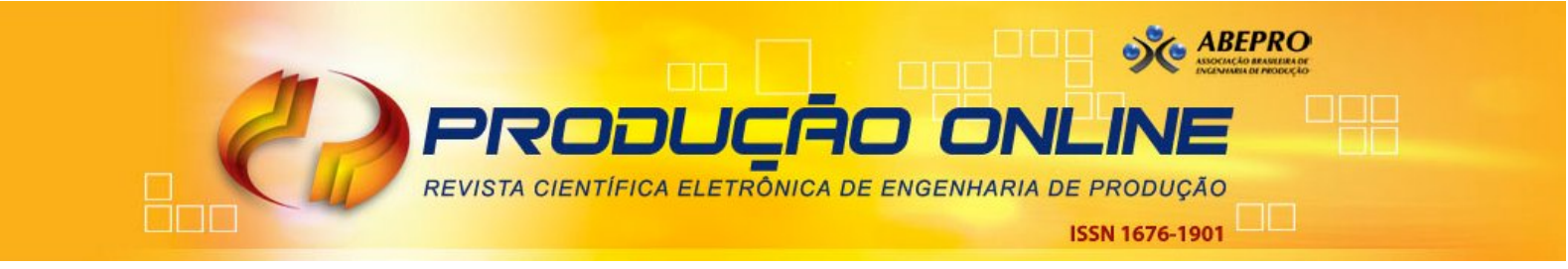

\title{
ABORDAGEM MULTICRITÉRIO DE APOIO A DECISÕES ESTRATEGICAMENTE SUSTENTÁVEIS NAS ORGANIZAÇÕES
}

\section{MULTI-CRITERIA APPROACH TO SUPPORT STRATEGICALLY SUSTAINABLE DECISIONS IN ORGANIZATIONS}

\author{
Victor Diogho Heuer de Carvalho*E-mail: victorheuer@gmail.com \\ Thiago Poleto* E-mail: thiagopoleto@hotmail.com \\ Lucio Camara e Silva* E-mail: lucio camara@hotmail.com \\ Ana Paula Cabral Seixas Costa* E-mail: apcabral@hotmail.com \\ *Universidade Federal de Pernambuco (UFPE), Recife, PE
}

\begin{abstract}
Resumo: Manter as condições socioambientais em um estado de conservação favorável para que a sociedade se desenvolva sem restrições é a ideia que embasa o conceito de Sustentabilidade. Diversas esferas como as mídias, a academia e as organizações passaram a dar maior importância para o estudo da sustentabilidade, uma vez que ela impacta diretamente em aspectos que influenciam a economia no mundo. Organizacionalmente, o uso de estratégias sustentáveis é capaz de trazer retornos em prol de um desenvolvimento saudável e de uma cultura consciente quanto aos desperdícios, além de refletir na forma como as organizações mantém seu relacionamento com a sociedade e o meio ambiente. O objetivo deste trabalho é propor um modelo para a priorização de estratégias que apoiem a criação de um ambiente e uma cultura baseados em sustentabilidade nas organizações. Tal modelo possui quatro etapas, iniciando-se com a estruturação de problemas, passando pela definição de critérios e alternativas, aplicação de método multicritério para priorização e, por fim, chegando à implementação das alternativas de acordo com o que o decisor julgue adequado, tomando por base o resultado da priorização.
\end{abstract}

Palavras-chave: Sustentabilidade. Estratégias Sustentáveis. PROMETHEE II.

Abstract: Keeping the social and environmental conditions in a favorable conservation status for allowing the society to develop itself without restrictions is the central idea which underpins the sustainability concept. Different spheres such as media, academia and business organizations started to give more importance to the study of sustainability, once it directly affects aspects that influence the economy in the world. Organizationally, the use of sustainable strategies can bring returns in favor of a healthy development and a conscious culture about the waste as well as reflecting how organizations connect with society and the environment. The objective of this work is to propose a model for prioritizing strategies to support the creation of an environment and a culture based on sustainability in organizations. This model has four steps, beginning with the problem structuring, including the definition of criteria and alternatives, the application of a multi-criteria method for prioritization, and finally, the implementation of the alternatives according to the decision maker opinion as well as on prioritization results.

Keywords: Sustainability. Sustainable Strategies. PROMETHEE II.

\section{INTRODUÇÃO}

Diversas áreas passaram a definir maior importância para a questão da sustentabilidade, seja na esfera empresarial, governamental ou mesmo na 
sociedade em geral. Dentro do contexto empresarial, vários estudiosos vêm desenvolvendo análises que levam em conta a imersão da sustentabilidade com o objetivo de criar uma cultura organizacional sustentável. Nestes estudos são trabalhadas questões-chave como a difusão organizacional do conceito de sustentabilidade e o motivo pelo qual as empresas variam tanto em opinião para justificar a adoção de medidas sustentáveis, considerando ainda a influência que cada tipo de cultura tem sobre as decisões relacionadas (CAPRAR e NEVILLE, 2012).

O contexto governamental considera o poder das autoridades e governos locais como elemento preponderante para a criação de iniciativas sustentáveis nas organizações. Neste ponto de vista estão incluídos o controle e a regulamentação de diversos elementos como o consumo de energia e de água, os índices de poluição, uso de recursos naturais e da terra (GLASS, 2002; COYLE, 2012).

No que diz respeito a atuação da sociedade, ela é marcada por constantes mudanças no uso de tecnologias que exercem influência sobre a cultura das massas e sobre as práticas e costumes a nível pessoal, o que caracteriza a sociedade como um sistema em contínua evolução. A forma como as pessoas pensam sobre a importância do meio ambiente em seus lares e no trabalho é também um resultado desta influência (SAFARZYŃSKA, 2012).

A emergência dessas questões fez com que a Comissão Mundial sobre Meio Ambiente e Desenvolvimento (1991) conceituasse Desenvolvimento Sustentável como "um desenvolvimento que satisfaz as necessidades do presente sem comprometer a capacidade das gerações futuras satisfazerem suas próprias necessidades".

O Desenvolvimento Sustentável possui alguns elementos destacados, com maior incidência em termos de preocupação das autoridades e recorrência em pesquisas científicas. Os dois principais a serem mencionados são os efeitos da poluição, com a criação de medida para diminuí-los, e o consumo de energia (MAIA; PIRES, 2001; RASKA; SHAW, 2012).

Tukker (2013) também considerou a educação a nível escolar e organizacional para criação de uma cultura sustentável como um elemento de fundamental importância, uma vez que é a partir da educação que se pode criar uma mentalidade propícia para a definição de uma cultura sustentável. Cruz, Pedro e 
Estivalete (2006) citaram o emprego de políticas e estratégias sustentáveis envolvendo diversos níveis organizacionais como mais um elemento que exerce influência direta sobre a questão da criação de uma cultura sustentável.

Com base nestes diversos elementos e aspectos sobre sustentabilidade, Poleto et al. (2013) desenvolveram um mapeamento cognitivo com um grupo de decisores utilizando a metodologia de Desenvolvimento e Análise de Opções Estratégicas (Strategic Options Development and Analysis - SODA) para estruturar problemas relacionados com sustentabilidade, os quais podem ser enquadrados em quatro pontos de vista: Ambiental, Social, Econômico e Institucional. Os mapas criados contemplaram as seguintes áreas: Sustentabilidade da Produção, dos Produtos e dos Recursos.

O objetivo deste artigo é apresentar a proposta de uma abordagem multicritério baseada em priorização de alternativas que podem ser entendidas como estratégias em sustentabilidade para adoção nas organizações. O trabalho dá continuidade às pesquisas desenvolvidas por Poleto et al. (2013) e Carvalho et al. (2013), realizando uma junção dos procedimentos empregados em ambas além de expandi-las.

O restante deste artigo se divide da seguinte forma: a Seção 2 apresenta um referencial teórico sobre sustentabilidade e apoio a tomada de decisão; a Seção 3 apresenta o modelo de decisão aplicado, demonstrando como o problema foi estruturado e contendo a definição das alternativas e critérios para que seja realizada a priorização destas alternativas; por fim, a Seção 4 apresenta a conclusão deste trabalho com a proposta de trabalhos futuros para dar continuidade as ideias aqui apresentadas.

\section{REFERENCIAL TEÓRICO}

\subsection{Sustentabilidade como Estratégia Organizacional}

Com o conceito de Sustentabilidade vem a necessidade de se repensar o desenvolvimento econômico, trazendo à tona a ideia de uma Economia Verde (DINIZ e BERMAN, 2012). Por Economia Verde se entende a economia que resulta na melhoria do bem-estar humano e na equidade social, e ao mesmo tempo reduz 
os riscos ambientais e a escassez ecológica (UNITED NATIONS ENVIRONMENT PROGRAMME, 2011).

Para garantir a continuidade da ideia de Economia Verde, as organizações têm se preocupado em entender como o desenvolvimento sustentável deve suportar suas atividades, para que sejam criadas estratégias sustentáveis de negócios. Conforme Tukker (2013), atingir uma estratégia de negócios sustentável não é uma tarefa fácil, uma vez que envolve inúmeros trade-offs a serem julgados, assim como o entendimento de como a decisão tomada irá afetar o ambiente e a cultura organizacional.

Cronin Junior et al. (2011) utilizaram o conceito de estratégias de Marketing Verde, com ações nas áreas de Recursos Humanos, Marketing e Gestão de Operações (que os autores definem como as disciplinas centrais da Gestão de Negócios) de forma a determinar, junto aos stakeholders nas organizações, quais estratégias poderiam ser adotas e qual o efeito delas sobre eles próprios, além de identificar outras oportunidades de estudos na área.

Para determinação dessas estratégias, Connelly et al. (2011) realizaram um levantamento das principais temáticas a serem exploradas em pesquisas sobre sustentabilidade, identificando uma série de perspectivas teóricas: Custos de Transações Econômicas, Teoria da Agência, Teoria Institucional, Ecologia Organizacional, dentre outras. Os autores sugerem que a exploração destes assuntos em mais pesquisas sobre Sustentabilidade podem gerar entendimentos de alternativas que possam embasar os gestores na adoção de estratégias sustentáveis para suas organizações.

O efeito dessas alternativas pode ser verificado através do trabalho de Tachizawa (2009), que apresentou uma arquitetura de dados socioambientais para diagnóstico das organizações em termos de sustentabilidade econômica. Seu trabalho também propôs um modelo de base de dados de indicadores socioambientais, fundamentado em pesquisa empírica, cuja principal contribuição é o mapeamento socioambiental dos diferentes segmentos econômicos do universo empresarial brasileiro.

No âmbito do setor têxtil e de confecção, por exemplo, Bruno e Bruno (2009) discutiram os resultados das atividades desenvolvidas, confrontando-os com iniciativas de enfoque socioambiental e com tendências de consumo consciente no 
setor. A partir dos resultados foram elaboradas oficinas entre os envolvidos a fim de promover políticas de desenvolvimento sustentável que possam servir de referência para outras cadeias e atividades econômicas.

Por fim, Cruz et al. (2006) estabeleceram um framework para analisar a movimentação da estratégia organizacional sob a ótica baseada na lógica financeiraeconômica para a ótica baseada na lógica sustentável. Dentre as principais implicações dos achados desta pesquisa, constatou-se que há a necessidade de ações sustentáveis de negócios que sejam orientadas para a sociedade em geral, de forma que existem demandas dentro das organizações por responsabilidades socioambientais.

\subsection{Métodos de estruturação de problema}

A aplicação de Métodos de Estruturação de Problemas (Problem Structuring Methods - PSM) em processos de tomada de decisão em grupo em ambientes complexos e com diversos vieses é uma alternativa bastante viável uma vez que promove o trabalho conjunto de um analista com decisores para a obtenção de um esquema de como o problema, na visão destes decisores, pode ser representado (ACKERMANN, 2012).

$\mathrm{Na}$ literatura especializada destacam-se os seguintes métodos: Pensamento Focado em Valor (Value Focused Thinking - VFT), o já referido SODA, a Metodologia de Sistemas Suaves (Soft Systems Methodology - SSM) e a Abordagem de Escolhas Estratégicas (Strategic Choice Approach - SCA). Estes métodos provêm um melhor entendimento entre os decisores sobre os assuntos em que o problema se baseia (ALMEIDA et al., 2012).

O presente estudo, enquanto continuidade da pesquisa realizada por Poleto et al. (2013), utilizou o SODA, que possibilita a captura de pontos de vista, conhecimento e expertise envolvidas com o problema de decisão, através de entrevistas com pessoas chave para a resolução deste problema, ou através da análise de documentos de referência, sendo possível também a realização de entrevistas com pessoas especializadas na área em que o problema está enquadrado (EDEN, 2004). 
A metodologia do SODA tem como destaque a elaboração de mapas cognitivos que serão construídos pela exploração de cada contribuição individual capturada do grupo de decisores. Com isso, é estruturada uma rede onde as metas ou aspirações aparecem no topo dessa da estrutura e os elementos de suporte aparecendo abaixo (ACKERMANN e EDEN, 2001).

\subsection{Apoio Multicritério a Decisão}

A aplicação de uma abordagem multicritério exige o conhecimento de conceitos básicos como o de critérios, alternativas e pesos, de forma que as alternativas serão avaliadas segundo os múltiplos critérios definidos e cada critério também possui um peso atribuído pelo decisor.

Outro elemento importante, quando se trabalha com a abordagem multicritério, é a definição da problemática de decisão que irá auxiliar na determinação do método a ser utilizado para o apoio à decisão. Em termos de problemática Roy (1996) estabeleceu a seguinte tipologia: Problemática $P$. $\alpha$ ou de Escolha, Problemática P. $\beta$ ou de Classificação, Problemática P.Y ou de Ordenação, Problemática $P . \delta$ ou de Descrição.

Seguindo as definições de Carvalho et al. (2014), para o problema tratado neste trabalho, será utilizada problemática de ordenação, uma vez que se deseja obter uma priorização de alternativas em sustentabilidade, provendo ao decisor uma visualização da melhor alternativa para a pior.

Métodos de sobreclassificação (ou outranking) como o PROMETHEE II e o ELECTRE II foram criados com base na problemática de ordenação. Este trabalho considerou a aplicação do PROMETHEE II por ele estar bastante enquadrado com as particularidades do problema de decisão estabelecido, tendo como vantagem sobre o ELECTRE II o fato de ser mais sensível a pequenas modificações, possibilitando ainda um melhor entendimento dos resultados para os decisores (BRANS, 1982).

O procedimento do PROMETHEE II é também considerado mais complacente, já que basta uma alternativa ter um fluxo liquido maior que a outra para ter uma posição melhor na priorização. No ELECTRE II, por sua vez, é necessário que haja sobreclassificação conforme planos de corte estabelecidos 
através de níveis de concordância mínima e de discordância máxima (ALMEIDA, 2013).

Os métodos PROMETHEE foram criados por Brans (1982) e expandidos por trabalhos posteriores dele com outros autores, como ocorre em Brans e Vincke (1985). Os métodos que pertencem a esta família possuem duas fases principais: (i) a construção de uma relação de sobreclassificação com a agregação entre alternativas e entre critérios e (ii) a exploração das relações obtidas (BRANS e MARESCHAL, 2002).

A estrutura de avaliação dos métodos PROMETHEE é baseada na definição de pesos para todos os critérios, para que a partir destes pesos, possa-se obter o grau de sobreclassificação de uma alternativa em relação à outra, sendo isso realizado para cada par de alternativas (ALMEIDA, 2013). Assim, o método realiza um comparação par-a-par entre as alternativas.

O PROMETHEE II utiliza uma função de preferência $F(a, b)$ de a sobre $b$, que é definida pela Equação 1 (BRANS e VINCKE, 1985).

$$
F(a, b)=\left\{\begin{array}{c}
0 \quad \text { se } f(a) \leq f(b) \\
p[f(a), f(b)] \text { se } f(a)>f(b)
\end{array}\right.
$$

Aqui será considerada a função de preferência para critério usual, havendo assim só dois tipos de relação: preferência e indiferença. Tal fato simplifica a análise para o decisor, facilitando seu entendimento. A Equação 2 representa o tipo de função de preferência adotada.

$$
F(x)=\left\{\begin{array}{l}
0 \text { para todo } x \leq 0 \\
1 \text { para todo } x>0
\end{array}\right.
$$

O grau de sobreclassificação é representado por $\pi(a, b)$, sendo obtido através da aplicação da Equação 3.

$$
\pi(a, b)=\sum_{i=1}^{n} p_{i} F_{i}(a, b)
$$

Em que:

$\sum_{i=1}^{n} p_{i}=1$;

$\pi$ é o peso de cada critério;

$F_{i}(a, b)$ ré a função da diferença $\left[g_{i}(a)-g_{i}(b)\right]$ entre o desempenho das alternativas para cada critério $i$. 
A função da diferença terá valores entre 0 e 1 , onde 0 representa uma indiferença (I) e 1 uma preferência estrita $(P)$.

Com o PROMETHEE II é obtida uma pré-ordem completa indo desde a melhor alternativa até a pior delas. Para tanto é utilizado um fluxo líquido obtido por meio da diferença entre um fluxo positivo e um fluxo negativo. Este fluxo líquido representa o balanço entre a força e a fraqueza de cada alternativa (ALMEIDA, 2013).

As Equações 4, 5 e 6 a seguir representam os fluxos positivo, negativo e líquido, consecutivamente.

$$
\begin{gathered}
\Phi^{+}(a)=\sum_{b \in A} \pi(a, b) \\
\Phi^{-}(a)=\sum_{b \in A} \pi(b, a) \\
\Phi(a)=\Phi^{+}(a)+\Phi^{-}(a)
\end{gathered}
$$

A partir do indicador $\Phi(a)$, pode-se organizar as alternativas em ordem decrescente, ordenação obtida com base nas relações de Preferência ( $a P b)$ se $\Phi(a)$ $>\Phi(b)$ e Indiferença (alb) se $\Phi(a)<\Phi(b)$ (ALMEIDA, 2013). Brans e Vincke (1985), Brans e Mareschal (2002) e Behzadian et al. (2010) fornecem mais detalhes sobre o método PROMETHEE II.

\subsection{Aplicações Multicritério na Sustentabilidade}

A preocupação com o meio ambiente tem se tornado um dos principais desafios na atualidade e com ela houve a necessidade de um enquadramento à novas posturas e conscientização por parte de governos, consumidores, empresas e a sociedade como um todo.

Em geral, as grandes cidades apresentam um elevado índice de poluição ambiental em diferentes categorias, tais como consumo de recursos naturais, geração de resíduos sólidos, transporte e poluição, causando um impacto significativo sobre o meio ambiente. Nesse sentido, Egilmez et al. (2015) utilizaram a abordagem multicritério para mensurar o índice de poluição ambiental e demonstrar, 
dentre as principais cidades do Canadá, aquelas que tem o maior impacto sobre meio ambiente. Como uma das importantes contribuições de sua pesquisa, o julgamento de especialistas fornece informações significativas sobre a utilização de indicadores de sustentabilidade.

A indústria de petróleo tem um impacto significativo na poluição do meio ambiente. Desta forma, Rabbani et al. (2014) propuseram a avaliação do desempenho sustentável das as empresas produtoras de petróleo no Irã, através do uso do balanced scorecard (BSC) e da abordagem multicritério para essa avaliação foram utilizados critérios econômico, ambiental, social, processos internos, crescimento e aprendizado. A principal contribuição foi a realização de um diagnóstico das empresas quanto a sustentabilidade e prover um mapa estratégico para adoção de políticas para desenvolvimento futuro.

No contexto agroindustrial, a contaminação do solo é uma das principais ameaças que limitam a produtividade de diferentes produtos. Baseado neste problema, Volchko et al. (2014) propuseram o uso métodos multicritérios para avaliar a qualidade do solo, considerando os seguintes aspectos, a textura do solo, teor de matéria orgânica, capacidade de agua disponível, $\mathrm{PH}$, fosforo disponível e, nitrogênio mineral. A proposta de Volchko et al. (2014) foi a redução dos riscos apresentados pelos contaminantes para a saúde humana e para o meio ambiente.

Silva e Costa (2011) apresentaram um modelo de apoio a decisão multicritério para auxiliar o processo de tomada de decisão na adoção de políticas de sustentabilidade nas empresas de tecnologia. Para isto, foi aplicado o SODA para estruturar o problema, a fim de alcançar benefícios estratégicos.

Quanto ao uso de fontes de energia renováveis, Mourmouris e Potolias (2013) utilizaram uma abordagem de decisão multicritério a fim de explorar o potencial de energia eólica, solar, biomassa, geotérmica e pequenas centrais hidrelétricas, para geração de calor. A proposta de Mourmouris e Potolias (2013) foi descobrir a quantidade ideal de cada fonte de energia renovável que pode ser produzido na região.

Portanto, a abordagem central dos trabalhos mencionados auxilia na justificativa da utilização de métodos multicritério para apoiar a tomada de decisão sobre o uso de políticas e estratégias de sustentabilidade nas organizações, 
permitindo ao decisor uma visão mais estruturada de acordo com uma determinada problemática sobre a qual o problema de decisão foi formulado.

No contexto de sustentabilidade, os custos para implementação são altos, sendo assim, nem sempre as empresas possuem recursos financeiros para implementar todas as políticas ao mesmo tempo, desta forma, a contribuição deste trabalho é auxiliar os gestores a decidir sobre quais políticas de sustentabilidades deverão ser implementadas nas empresas.

\section{MODELO DE DECISÃO PARA PRIORIZAR ESTRATÉGIAS DE SUSTENTABILIDADE}

O modelo de decisão proposto nesse trabalho é baseado em 4 etapas e tem por objetivo priorizar alternativas para resolução de problemas de sustentabilidade dentro das organizações. A Figura 1 representa as etapas deste modelo.

Figura 1 - Etapas do modelo proposto para priorizar as políticas de sustentabilidade.

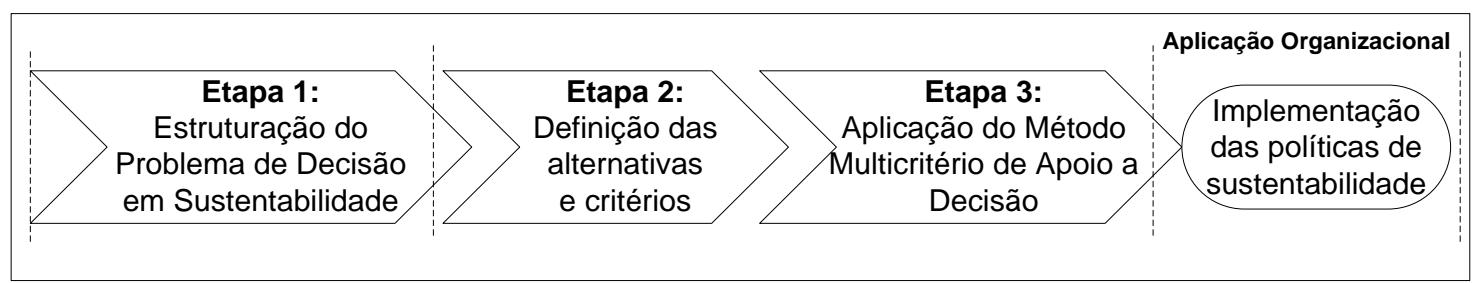

Fonte: Autor (2014)

A etapa 1 do modelo corresponde a fase de estruturação do problema, baseada na proposta de Poleto et al. (2013). Através de uma revisão da literatura, foram obtidos os critérios de avaliação e através dos mapas cognitivos gerados através do SODA foram estabelecidas as alternativas, de forma que estas definições dizem respeito à etapa 2 do modelo.

A etapa 3 se refere a aplicação do método multicritério, de acordo com o que foi assumido por Carvalho et al. (2014). Como mencionado anteriormente, estes autores consideraram a problemática de ordenação com a aplicação do PROMETHEE II, fornecendo uma priorização para os decisores escolherem quais as políticas ou estratégias em sustentabilidade a serem implementadas. A etapa final de aplicação organizacional, consiste na própria implementação das alternativas escolhidas a nível organizacional. 


\subsection{Estruturação do Problema de Sustentabilidade}

A elaboração dos mapas cognitivos se inicia a partir do pressuposto de que a organização não obtém a percepção clara da sustentabilidade, considerando uma visão global da sustentabilidade de produtos, produção e recursos no seu processo. Assim, novas perspectivas foram criadas, alinhadas com os objetivos desejados pela organização. É relevante destacar também que o mapa cognitivo não se trata de um modelo de decisão, mas de uma forma de auxiliar o decisor a pensar de uma maneira mais estruturada sobre o problema.

Os mapas gerados com a aplicação do SODA e que foram aplicados para embasar este estudo, representam estruturas cognitivas dos decisores sobre aspectos organizacionais da sustentabilidade, sendo corroborados por várias pesquisas (FADZIL et al., 2012; HEIKKURINENA e BONNEDAHL, 2013; RASKA e SHAW, 2012). Os conceitos dispostos nos mapas expressam os entendimentos, explicações e estratégias que refletem, de modo geral, a questão da sustentabilidade. As ligações entre esses conceitos são representadas por setas, as quais indicam como um conceito conduz ou tem implicação sobre outro.

A visão global da sustentabilidade nas organizações gerou três mapas cognitivos que demonstram diferentes perspectivas de sustentabilidade, de forma que estes três mapas foram sintetizados em um mapa sumário conforme apresentado na Figuras 2. Este mapa expressa as representações discursivas baseadas na percepção de um decisor sob uma determinada questão, dando suporte ao mesmo na adoção de políticas e/ou ações estratégicas em prol da sustentabilidade. 
Figura 2 - Mapa Cognitivo sumário obtido através do SODA.

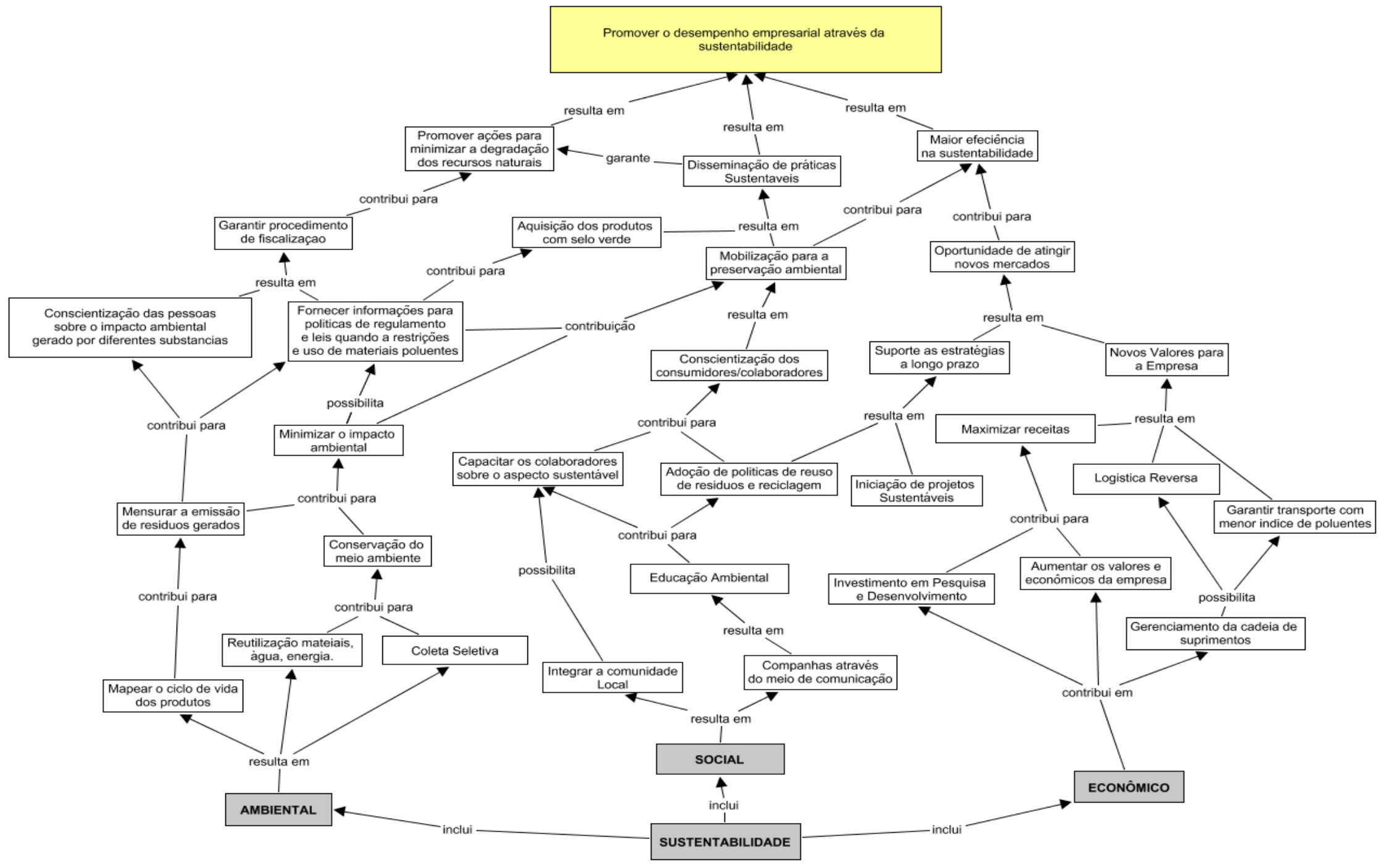

Fonte: Autor (2015) 


\subsection{Identificação das Alternativas e dos Critérios}

Com da elaboração dos mapas cognitivos foi possível realizar algumas deduções sobre o problema de decisão. Estas deduções tiveram por base as ideias e opiniões dos decisores para a geração de ações em estratégias de sustentabilidade.

Tomando por base também as definições de Poleto et al. (2013) e Carvalho et al. (2014), foram determinados conjuntos de ações em sustentabilidade entendidos como agrupamentos de alternativas para uso no método PROMETHEE II. Sendo assim, existem três grandes conjuntos de ações para cada área, que são: um voltado para sustentabilidade da produção, outro para sustentabilidade de produtos e outro dos recursos.

O Quadro 1 demonstra as alternativas obtidas para cada conjunto de ações.

Quadro 1 - Conjuntos de Ações e suas Alternativas

\begin{tabular}{|c|c|}
\hline Conjuntos de Ações & Alternativas \\
\hline \multirow{4}{*}{$\begin{array}{c}\text { Ações em Sustentabilidade de } \\
\text { Produtos }\end{array}$} & (A1) Educação e Conscientização Ambiental. \\
\hline & (A2) Reuso de Materiais e Recursos. \\
\hline & (A3) Aquisição de produtos com selo verde. \\
\hline & (A4) Investimento em P\&D sustentável. \\
\hline \multirow{5}{*}{$\begin{array}{c}\text { Ações em Sustentabilidade da } \\
\text { Produção }\end{array}$} & $\begin{array}{l}\text { (A5) Controle de Poluentes (gases, resíduos sólidos e } \\
\text { líquidos). }\end{array}$ \\
\hline & (A6) Uso de fontes de energia renováveis. \\
\hline & (A7) Reaproveitamento e controle da água. \\
\hline & (A8) Uso de Logística reversa. \\
\hline & (A9) Contratação de mão-de-obra local. \\
\hline \multirow{3}{*}{$\begin{array}{c}\text { Ações em Sustentabilidade de } \\
\text { Recursos }\end{array}$} & (A10) Implementação de Corredores Ecológicos. \\
\hline & (A11) Transformação de resíduos em produtos para venda. \\
\hline & (A12) Reaproveitamento de matérias-primas. \\
\hline
\end{tabular}

Fonte: Carvalho et al. (2014)

A seguir será realizada a descrição de cada uma das alternativas conforme opiniões levantadas junto aos decisores em paralelo a aplicação do SODA:

- A1 - Educação e Conscientização Ambiental: a Educação Ambiental tem por objetivo promover a mudança cultural em relação ao meio ambiente, de forma a garantir a melhoria da qualidade de vida dentro e fora das organizações. Ela possibilita a empresa melhorias sobre a sustentabilidade, auxiliando na criação de uma nova mentalidade relacionada a como usufruir dos recursos oferecidos pela natureza. 
- A2 - Reuso de Materiais e Recursos: trata-se do reaproveitamento de materiais que não passaram por qualquer tipo de processamento. Os materiais utilizados na produção podem ter ainda muitos usos sem a necessidade um processo de reciclagem. Isso possibilita para a empresa melhorias sobre o aspecto de criação e inovação, baseadas no reaproveitamento de um produto.

- A3 - Aquisição de produtos com selo verde: os selos conferem aos produtos a constatação de que eles passaram por avaliações de conformidade e qualidade de acordo com critérios e normas nacionais e internacionais. Possibilita a empresa incrementar as vendas e agrega valor à imagem do produto.

- A4 - Investimento em P\&D sustentável: consiste em investir em pesquisas no meio empresarial que auxiliem no desenvolvimento de produtos sustentáveis. Esta alternativa auxilia no fortalecimento da imagem da empresa em relação a sua preocupação com o meio ambiente.

- A5 - Controle de Poluentes (gases, resíduos sólidos e líquidos): poluição é qualquer alteração no meio-ambiente, trazendo prejuízos ao homem e às outras formas de vida que nele habitam. A proposta para as empresas é adotar medidas de controle, proporcionando um aumento na conscientização dos indivíduos, além de minimizar o impacto no meio ambiente.

- A6 - Uso de energias renováveis: energias renováveis são aquelas que vêm de fontes que não cessão, tais quais o vento (energia eólica), a água (energia hídrica ou hidráulica) ou o sol (energia solar). Ao contrário dos combustíveis não-renováveis (como os de origem fóssil). Geralmente causam um pouco impacto ao meio ambiente.

- A7 - Controle do uso da água: diz respeito ao controle de desperdício da água, de forma a garantir que haja água para consumo humano seja na alimentação ou para uso no saneamento e na irrigação. Essa medida auxilia também na redução dos custos envolvidos e na manutenção do nível ótimo de água nos grandes reservatórios que alimentam regiões urbanas e rurais. 
- A8 - Uso da logística reversa: esta medida possibilita que resíduos sólidos retornem para suas empresas de origem, evitando seu descarte de forma inadequada e que cause poluição. Além disso, é capaz de garantir economias para o processo produtivo já que estes resíduos retornam para a cadeia produtiva.

- $\quad$ A9 - Contratação de mão-de-obra local: a contratação de mão-de-obra local possibilita maior movimentação na economia regional e, além disso, aumenta a empregabilidade.

- A10 - Implementação de Corredores Ecológicos: corredores ecológicos são áreas verdes implantadas pelas empresas que visam a preservação ecológica, sendo destacadas como medidas de reflorestamento e conservação da biodiversidade local.

- A11 - Transformação de resíduos sólidos para venda: é um processo de transformação dos resíduos sólidos envolvendo alterações físicoquímicas que criem novos materiais para produção de novos produtos. Entre as grandes vantagens desse processo podem ser citadas: economia de recursos naturais, redução da necessidade de extração de minérios em minas e de seus impactos ambientais, aumento de economia da empresa com rendas alternativas, etc.

- A12 - Reaproveitamento de matéria-prima: reuso de alguns materiais utilizados em produções anteriores por meio de reprocessamento ou aproveitamento de sobras utilizáveis. Trata-se de uma alternativa capaz de gerar ganhos operacionais uma vez que reduz o consumo de matérias-primas.

$\mathrm{Na}$ Etapa 2 do modelo proposto foi realizada uma revisão da literatura sobre sustentabilidade para definição dos critérios. Os seguintes critérios foram definidos: grau de impacto técnico, grau de impacto financeiro do investimento, grau de impacto social e grau de impacto nas estratégias de negócios. O Quadro 2 apresenta estes critérios e respectivos conceitos com os autores que os mencionam. 
Quadro 2 - Conjunto de critérios e suas definições

\begin{tabular}{|c|c|c|}
\hline Critérios & Definição & Referencial \\
\hline $\begin{array}{l}\text { (C1) - Grau de } \\
\text { impacto financeiro }\end{array}$ & $\begin{array}{l}\text { Impacto positivo na organização do } \\
\text { retorno financeiro - ganho ou } \\
\text { benefício financeiro - para um } \\
\text { investimento em determinada } \\
\text { alternativa. }\end{array}$ & $\begin{array}{l}\text { Afgan e Carvalho (2002), } \\
\text { Becalli et al. (2003), Huang et } \\
\text { al. (2005), Doukas et al. (2007), } \\
\text { Wang et al. (2008), } \\
\text { Papadopoulos e Karagiannidis } \\
\text { (2008) e Javanovich et al. } \\
(2009) \text {. }\end{array}$ \\
\hline $\begin{array}{l}\text { (C2) - Grau } \\
\text { Impacto Social }\end{array}$ & $\begin{array}{l}\text { Impacto social positivo de } \\
\text { determinada alternativa em prol do } \\
\text { desenvolvimento social sustentável } \\
\text { local, considerando tanto o } \\
\text { ambiente interno quanto externo da } \\
\text { organização aumentando o nível de } \\
\text { bem-estar social. }\end{array}$ & 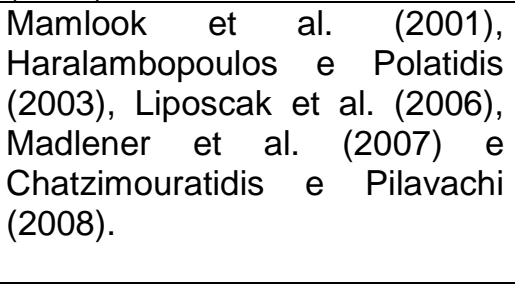 \\
\hline $\begin{array}{lll}\text { (C3) - } & \text { Grau } & \text { de } \\
\text { impacto } & & \text { na } \\
\text { Estratégia } & & \text { de } \\
\text { Negócio } & & \\
& & \end{array}$ & $\begin{array}{l}\text { Impacto positivo que a alternativa } \\
\text { pode ocasionar de forma a auxiliar } \\
\text { na melhoria da estratégia de } \\
\text { negócio da organização, } \\
\text { aumentando sua visibilidade no } \\
\text { mercado e na sociedade. }\end{array}$ & Lin \\
\hline $\begin{array}{lr}\mathrm{C} 4) \quad-\quad \text { Grau } & \text { de } \\
\text { Impacto Técnico } & \end{array}$ & $\begin{array}{l}\text { Impacto positivo do emprego de } \\
\text { determinada alternativa de } \\
\text { sustentabilidade em aspectos } \\
\text { técnicos e operacionais de } \\
\text { organização, maximizando a } \\
\text { utilização dos recursos e buscando } \\
\text { minimizar o impacto ambiental do } \\
\text { uso destes recursos. }\end{array}$ & $\begin{array}{l}\text { Mamlook et al. (2001), Afgan e } \\
\text { Carvalho (2002), Doukas et al. } \\
\text { (2007), Chatzimoratidis et al. } \\
\text { (2008), Jovanovic et al. (2009), } \\
\text { Pilavachi et al. (2009) e Wang } \\
\text { et al. (2008). }\end{array}$ \\
\hline
\end{tabular}

Fonte: Carvalho et al. (2014)

\subsection{Aplicação do PROMETHEE II para Priorização das Alternativas}

Estruturado o problema de decisão, pode-se partir para a Etapa 3, que se iniciou com a agregação de preferências do método PROMETHEE II.

Foi utilizado um decisor especialista em gestão empresarial que avaliou as alternativas através dos critérios estabelecidos. O especialista também definiu os pesos dos critérios (suas importâncias relativas) da seguinte forma: C1 teve peso 0,30; ao C2 foi atribuído peso 0,20; para C3 o peso determinado foi 0,35; e por fim, foi definido o peso de 0,15 para C4.

Esta avaliação foi feita de forma direta junto ao decisor, embora existam métodos formais para realizar a elicitação destes pesos, caso o decisor tenha dificuldades para atribuir importâncias aos critérios.

A avaliação das alternativas foi realizada através de uma escala de Likert de 5 pontos, onde 1 representa um baixo impacto e 5 um alto impacto. A Tabela 1 apresenta a avaliação deste decisor. 
Tabela 1 - Matriz de avaliação das alternativas de acordo com os critérios

\begin{tabular}{lrrrr}
\hline \multicolumn{1}{c}{ Políticas de } & \multicolumn{4}{c}{ Critérios } \\
\cline { 2 - 5 } & C1 & C2 & C3 & C4 \\
\hline A1- Educação e Conscientização Ambilidade & 2 & 4 & 5 & 4 \\
A2 - Reuso de Materiais e Recursos & 2 & 2 & 4 & 5 \\
A3 - Aquisição de produtos com selo verde & 4 & 5 & 3 & 3 \\
A4 - Investimento em P\&D sustentável & 4 & 5 & 5 & 5 \\
A5 - Controle de Poluentes (gases, resíduos sólidos e líquidos) & 3 & 5 & 3 & 3 \\
A6 - Uso de fontes de energia renováveis & 4 & 4 & 4 & 5 \\
A7 - Reaproveitamento e controle da água. & 3 & 5 & 2 & 4 \\
A8 - Uso de Logística reversa & 3 & 4 & 5 & 5 \\
A9 - Contratação de mão de obra local & 3 & 4 & 1 & 4 \\
A10 - Implementação de Corredores Ecológicos & 5 & 4 & 1 & 3 \\
A11 - Transformação de resíduos em produtos para venda & 3 & 4 & 4 & 4 \\
A12 - Reaproveitamento de matérias-primas & 1 & 4 & 4 & 4 \\
\hline
\end{tabular}

Fonte: Autor (2015)

A partir desta avaliação, seguindo com o procedimento do PROMETHEE II, foram determinados os fluxos positivo, negativo e líquido. O fluxo líquido possibilitou o ordenamento das alternativas para a criação de seu ranking final. A Tabela 2 apresenta o ranking e os valores dos fluxos líquidos das alternativas.

Tabela 2 - Ranking das alternativas e os fluxos do PROMETHEE II

\begin{tabular}{ccccc}
\hline Posição & Alternativa & $\mathbf{\Phi}$ & $\boldsymbol{\Phi}^{+}$ & $\boldsymbol{\Phi}^{-}$ \\
\hline 1 & A1 & 0,5000 & 0,6227 & 0,1227 \\
2 & A8 & 0,4318 & 0,5682 & 0,1364 \\
3 & A12 & 0,3591 & 0,5182 & 0,1591 \\
4 & A2 & 0,2364 & 0,5273 & 0,2909 \\
5 & A11 & 0,0864 & 0,3273 & 0,2409 \\
6 & A6 & $-0,0091$ & 0,3273 & 0,3364 \\
7 & A7 & $-0,0318$ & 0,3773 & 0,4091 \\
8 & A5 & $-0,0591$ & 0,3682 & 0,4273 \\
9 & A4 & $-0,1318$ & 0,2455 & 0,3773 \\
10 & A3 & $-0,2773$ & 0,2864 & 0,5636 \\
11 & A9 & $-0,3273$ & 0,1682 & 0,4955 \\
12 & A10 & $-0,7773$ & 0,0182 & 0,7955 \\
\hline
\end{tabular}

Fonte: Autor (2015)

Com a agregação de preferências do decisor, foi possível determinar que A1 é a melhor alternativa seguida por A8. A alternativa A10 foi considerada a pior opção. Apresentados estes resultados ao decisor, o mesmo concordou que as Alternativas A1 e A8 teriam forte impacto positivo geral em sua organização, demonstrando inclusive que A8 poderia influenciar diretamente na posterior aplicação de outras alternativas relacionadas, como por exemplo A2 e A11.

O decisor definiu que a alternativa A1 possui alto impacto em toda a organização além de ter grande reflexo na sociedade, uma vez que a educação é a principal responsável por promover uma cultura em prol da sustentabilidade. 
Finalizando a aplicação do método, foi realizada uma simulação referente a diferenciação de pesos dos critérios para mudança de cenários, aplicando-se uma variação de $-10 \%$ para os critérios com os maiores pesos e $+10 \%$ para os critérios com os menores pesos. Com as mudanças de pesos, houve uma reversão de ordem entre as alternativas A1 e A8 e entre as alternativas A7 e A11.

\section{CONCLUSÕES}

Este trabalho teve por objetivo a identificação de alternativas em prol da sustentabilidade. Foi aplicada uma abordagem multicritério de apoio a decisão para priorizar as atividades que serão executadas em primeira instancia, dado que as organizações põem não possuir recursos financeiros suficientes para aplicar todas as opções estratégicas disponíveis.

Para a identificação das alternativas foi realizada uma análise dos mapas cognitivos gerados por Poleto et al., (2013). As alternativas puderam ser organizadas de acordo com conjuntos de ações obtidos através de um modelo de relação entre áreas de sustentabilidade que englobam os pontos de vista social, econômico, institucional e ambiental.

Após a identificação destas alternativas, foram definidos critérios relacionados aos aspectos financeiro, social, organizacional e técnico, para avaliação especifica de seu grau de impacto de seus retornos sobre a organização. Utilizando um decisor especialista, foi realizada a avaliação, de forma a se obter por meio da aplicação do método de sobreclassificação PROMETHEE II, o ordenamento da melhor alterativa para a pior, seguindo as definições realizadas por Carvalho et al. (2014).

A partir da visualização do ranking contendo a referida ordenação o decisor estabeleceu que os resultados são concordantes com seu ponto de vista enquanto gestor, demonstrando a viabilidade da aplicação do método e ressaltando a possibilidade de implementação das melhores alternativas a nível organizacional. $\mathrm{O}$ decisor também definiu a importância da aplicação da alternativa melhor posicionada, uma vez que ela possui grande influência na criação de uma cultura sustentável nas organizações.

Ressalta-se que as alternativas ordenadas podem ser traduzidas como estratégias sustentáveis para aplicação nas organizações. A interpretação das 
alternativas como estratégias sugere que a gestão da organização define a sustentabilidade como algo importante e que é capaz de trazer retornos significativos para a própria organização e para a sociedade.

Como sugestões para trabalhos futuros, propõe-se o uso de uma modelagem multicritério envolvendo um grupo de decisores (Decisão em Grupo), de forma a concordar ainda mais com a aplicação da metodologia SODA, dada sua adequação para aplicação em processos de Decisão em Grupo para a estruturação de problemas.

A exploração mais aprofundada dos mapas cognitivos em busca de mais estratégias de sustentabilidade, assim como a busca por mais critérios para julgar essas políticas, também são deixadas como propostas, de forma a garantir aos gestores uma gama maior de opções para possíveis implementações nas organizações.

\section{REFERÊNCIAS}

ACKERMANN, F. \& EDEN, C. Contrasting single user and networked group decision support systems for strategy making. Group Decision and Negotiation, v. 10, n. 1, p. 47-66, 2001. http://dx.doi.org/10.1023/A:1008708912048

ACKERMANN, F. Problem structuring methods 'in the Dock': Arguing the case for soft OR. European Journal of Operational Research, v. 219, n. 3, p. 652-58, 2012. http://dx.doi.org/10.1016/i.ejor.2011.11.014

AFGAN, N.H. \& CARVALHO, M.G. Multi-criteria assessment of new and renewable energy power plants. Energy, v. 27, n. 8, p. 739-755, 2002. http://dx.doi.org/10.1016/S03605442(02)00019-1

ALMEIDA, A. T. Processo de decisão nas organizações: construindo modelos de decisão multicritério. São Paulo: Atlas, 2013.

ALMEIDA A. T.; MORAIS, D. C.; COSTA, A. P. C. S.; ALENCAR, L. H. \& DAHER, S.F.D., Decisão em grupo e negociação: métodos e aplicações, São Paulo: Atlas, 2012.

BECCALI, M.; CELLURA, M. \& MISTRETTA, M. Decision-making in energy planning. Application of the Electre method at regional level for the diffusion of renewable energy technology. Renewable Energy, v. 28, n. 13, p. 2063-2087, 2003. http://dx.doi.org/10.1016/S0960-1481(03)00102-2

BEHZADIAN, M.; KAZEMZADEH, R. B.; ALBADVI, A. \& AGHDASI, M. PROMETHEE: A comprehensive literature review on methodologies and applications. European Journal of Operational Research, v. 200, p. 198-215, 2010. http://dx.doi.org/10.1016/i.ejor.2009.01.021

BRANS, J. P. L'ingenierie de la decision. Elaboration d'instruments d'aide a la decision. Methode PROMETHEE. In: NADEAU, R.; LANDRY, M. (Eds.). L'aide a la Decision: Nature, 
Instruments et Perspectives D'avenir. Quebec: Presses de Universite Laval, p. 183-214, 1982.

BRANS, J. P. \& MARESCHAL, B. Prométhée-Gaia: une méthodologie d'aide à la décision en présence de critères multiples. Bruxelles: Editions de l'Université de Bruxelles, 2002.

BRANS, J. P. \& VINCKE, P. A Preference Ranking Organisation Method: The PROMETHEE Method for Multiple Criteria Decision-Making. Management Science, v. 31, p. 647-656, 1985.

BRUNO, F. S. \& BRUNO, A. C. M. O papel do setor têxtil e de confecção brasileiro na liderança de um modelo sustentável de desenvolvimento. Revista Produção Online, Florianópolis, v. 9, n. 3, p. 551-572, 2009. http://dx.doi.org/10.14488/1676-1901.v9i3.319

CAPRAR, D. V. \& NEVILLE, B. A. "Norming" and "Conforming": Integrating Cultural and Institutional Explanations for Sustainability Adoption in Business. Journal of Business Ethics, v. 10, n. 2, p. 231-245, 2012. http://dx.doi.org/10.1007/s10551-012-1424-1

CARVALHO, V. D. H., POLETO, T., CAMARA, L. S. \& COSTA, A. P. C. Aplicação e Modelagem Multicritério para Priorização de Políticas visando a Sustentabilidade nas Organizações. In: ENCONTRO NACIONAL DE ENGENHARIA DE PRODUÇÃO, 34., 2014. Anais... Curitiba, 2014.

COYLE, I. M. A local Government Practitioner's view of the sustainability of defined benefit pension plans. Public Administration Review, v. 72, n.6, p. 786-787, 2012. http://dx.doi.org/10.1111/.1540-6210.2012.02663.x

CHATZIMOURATIDIS, A.I. \& PILAVACHI, P.A. Multicriteria evaluation of power plants impact on the living standard using the analytic hierarchy process. Energy Policy, v. 36, n. 3, p. 1074-1089, 2008. http://dx.doi.org/10.1016/j.enpol.2007.11.028

CHATZIMOURATIDIS, A.I. \& PILAVACHI, P.A. Sensitivity analysis of the evaluation of power plants impact on the living standard using the analytic hierarchy process. Energy Conversion and Management, v. 49, n. 12, p. 3599-3611, 2008.

http://dx.doi.org/10.1016/j.enconman.2008.07.009

COMISSÃO MUNDIAL SOBRE MEIO AMBIENTE E DESENVOLVIMENTO. Relatório Brundtland: nosso futuro em comum. 2. ed. Rio de Janeiro: Editora da Fundação Getúlio Vargas, 1991.

CONNELLY, B. L.; KETCHEN JUNIOR, D. J. \& SLATER, S. F. Toward a "theoretical toolbox" for sustainability research in marketing. Journal of the Academy of the Marketing Science, v. 39, n. 1, p. 86-100, 2011. http://dx.doi.org/10.1007/s11747-010-0199-0

CRONIN JUNIOR, J. J.; SMITH, J. S.; GLEIM, M. R.; RAMIREZ, E. \& MATINEZ, J. D. Green marketing strategies: an examination of stakeholders and the opportunities they present. Journal of the Academy of the Marketing Science, v. 39, n. 1, pp. 158-174, 2011. http://dx.doi.org/10.1007/s11747-010-0227-0

CRUZ, L. B.; PEDROZO, E. A. \& ESTIVALETE, V. F. B. Towards sustainable development strategies: A complex view following the contribution of Edgar Morin. Management Decision, v. 44, n. 7, p. 871-891, 2006. http://dx.doi.org/10.1108/00251740610680578 
DOUKAS, H.C.; ANDREAS, B.M. \& PSARRAS, J.E. Multi-criteria decision aid for the formulation of sustainable technological energy priorities using linguistic variables. European Journal of Operational Research, v. 182, n. 2, p. 844-855, 2007. http://dx.doi.org/10.1016/i.ejor.2006.08.037

EDEN, C. Analyzing cognitive maps to help structure issues or problems. European Journal of Operational Research, v. 159, n.3, p.673-689, 2006. http://dx.doi.org/10.1016/S03772217(03)00431-4

EGILMEZ, G.; GUMUS, S. \& KUCUKVAR, M. Environmental sustainability benchmarking of the U.S. and Canada metropoles: An expert judgment-based multi-criteria decision making approach. Cities, v. 42, Part A, p. 31-41, 2015. http://dx.doi.org/10.1016/j.cities.2014.08.006

GLASS, S. M. Sustainability and Local Government. Local Environment, v. 7, n. 1, p. 97102, 2002. http://dx.doi.org/10.1080/13549830220115448

FADZIL, Z. F.; HASHIM, H. S.; CHEANI, A. I. \& AZIZ, A. Developing a campus sustainability assessment framework for the National University of Malaysia. World Academy of Science, Engineering and Technology, v. 66, p.751-755, 2012.

HARALAMBOPOULOS, D.A. \& POLATIDIS, H. Renewable energy projects: structuring a multi-criteria group decision-making framework. Renewable Energy, v. 28, n. 6, pp. 961973, 2003. http://dx.doi.org/10.1016/S0960-1481(02)00072-1

HEIKKURINEN, P. \& BONNEDAHL K. J. Corporate responsibility for sustainable development: a review and conceptual comparison of market- and stakeholder-oriented strategies. Journal of Cleaner Production, v. 43, p. 191-198, 2013. http://dx.doi.org/10.1016/j.jclepro.2012.12.021

HUANG, F. Y.; WU, J.; WANG, R.Z. \& HUANG, X.H. Study on comprehensive evaluation model for combined cooling heating and power system (CCHP). Journal of Engineering Thermophysics, v. 26, p.13-16, 2005.

JOVANOVIC, M.; AFGAN, N.; RADOVANOVIC, P. \& STEVANOVIC, V. Sustainable development of the Belgrade energy system. Energy, v. 34, n. 5, p. 532-539, 2009. http://dx.doi.org/10.1016/j.energy.2008.01.013

LIPOSCAK, M.; AFGAN, N.H.; DUIC, N. \& CARVALHO, M. G. Sustainability assessment of cogeneration sector development in Croatia. Energy, v. 31, n. 13, p. 2276-2284, 2006. http://dx.doi.org/10.1016/j.energy.2006.01.024

MADLENER, R.; KOWALSKI, K. \& STAGL, S. New ways for the integrated appraisal of national energy scenarios: the case of renewable energy use in Austria. Energy Policy, $v$. 35, n. 12, p. 6060-6074, 2007. http://dx.doi.org/10.1016/j.enpol.2007.08.015

MAIA, A. G. \& PIRES, P. S. Uma Compreensão da Sustentabilidade por Meio dos Níveis de Complexidade das Decisões Organizacionais. Revista de Administração Mackenzie, v. 12, n. 3, p. 177-206, 2011. http://dx.doi.org/10.1590/S1678-69712011000300008

MAMLOOK, R.; AKASH, B.A. \& NIJMEH, S. Fuzzy sets programming to perform evaluation of solar systems in Jordan. Energy Conversion and Management, v. 42, n. 14, p. 17171726, 2001. http://dx.doi.org/10.1016/S0196-8904(00)00152-7 
MERRICK, J. R. W.; GRABOWSKI, M.; AYYALASOMAYAJULA, P. \& HARRALD, J. R. Understanding Organizational Safety using Value Focused Thinking. Risk Analysis, v. 25, n. 4, p. 1029-1041 2005. http://dx.doi.org/10.1111/j.1539-6924.2005.00654.x

MOURMOURIS,J. C. \& POTOLIAS, C. A multi-criteria methodology for energy planning and developing renewable energy sources at a regional level: A case study Thassos, Greece, Energy Policy, v 52, p. 522-530, 2013. http://dx.doi.org/10.1016/i.enpol.2012.09.074

PAPADOPOULOS, A. \& KARAGIANNIDIS, A. Application of the multi-criteria analysis method Electre III for the optimisation of decentralised energy systems. Omega, v. 36, n. 5, p. 766-776, 2008. http://dx.doi.org/10.1016/j.omega.2006.01.004

PILAVACHI, P.A.; STEPHANIDIS, S.D.; PAPPAS, V.A. \& AFGAN, N.H. Multi-criteria evaluation of hydrogen and natural gas fuelled power plant technologies. Applied Thermal Engineering, v. 29, n. 11-12, p. 2228-2234, 2009.

http://dx.doi.org/10.1016/j.applthermaleng.2008.11.014

POLETO, T.; SILVA, L. C.; MOURA, J. A. \& COSTA, A. P. C. S. A Utilização de Mapas Cognitivos para Estruturação de Problemas sob a Perspectiva da Sustentabilidade. In: ENCONTRO NACIONAL DE ENGENHARIA DE PRODUÇÃO,33., 2013. Anais... Salvador, 2013.

RASKA, D. \& SHAW, D. When is going green good for company image? Management Research Review, v. 35, n. 3, p. 326-347, 2012.

http://dx.doi.org/10.1108/01409171211210190

RABBANI, A.; ZAMANI, M.; YAZDANI-CHAMZINI, A. \& ZAVADSKAS, E. K., Proposing a new integrated model based on sustainability balanced scorecard (SBSC) and MCDM approaches by using linguistic variables for the performance evaluation of oil producing companies. Expert Systems with Applications, V.41, n.16, p. 7316-7327, 2014. http://dx.doi.org/10.1016/j.eswa.2014.05.023

ROY, B. Multicriteria methodology for decision aiding. Kluwer Academic Publishers, 1996.

SAFARZYŃSKA, K.; FRENKEN, K. \& BERGH, J. C. J. M. Evolutionary theorizing and modeling of sustainability transitions. Research Policy, v. 41, n. 6, pp. 1011-1024, 2012. http://dx.doi.org/10.1016/j.respol.2011.10.014

SELART, M. \& JOHANSEN, S. T. Understanding the Role of Value-Focused Thinking in Idea Management. Creativity and Innovation Management, v. 2, n. 3, p. 196-206, 2011. http://dx.doi.org/10.1111/j.1467-8691.2011.00602.x

SILVA, L. C., \& COSTA, A. P. C. S. Modelagem Multicritério para aplicação de políticas de sustentabilidade nas empresas de Tecnologia. In: SIMPÓSIO BRASILEIRO DE PESQUISA OPERACIONAL, 43., 2011. Anais... Ubatuba-SP 15- Agosto, 2011.

TACHIZAWA T. Sustentabilidade e responsabilidade social: proposta de modelo de diagnóstico socioambiental baseada em pesquisa empírica. Revista Produção Online, v. 9, n. 4, 2009. http://dx.doi.org/10.14488/1676-1901.v9i4.281

TUKKER, A. Knowledge collaboration and learning by aligning global sustainability programs: reflections in the context of Rio+20. Journal of Cleaner Production, v. 48, p. 272-279, 2013. http://dx.doi.org/10.1016/j.jclepro.2012.12.023 
UNITED NATIONS ENVIRONMENT PROGRAMME. Towards a green economy: pathways to sustainable development and poverty eradication - a synthesis for policy makers. 2011. Disponível em: < http://www.unep.org/greeneconomy/Portals/88/documents/ger/GER synthesis en.pdf>. Acessado em: 24 de Março de 2014.

WANG, J. J.; JING, Y.Y.; ZHANG, C.F.; SHI, G-H. \& ZHANG, X. T. A fuzzy multi-criteria decision-making model for trigeneration system. Energy Policy, v. 36, n. 10, p. 3823-3832, 2008. http://dx.doi.org/10.1016/i.enpol.2008.07.002

VOLCHKO, Y.; NORRMAN, J.; ROSÉN, L.; BERGKNUT, M.; JOSEFSSON, S.; SÖDERQVIST, T.; NORBERG, T.; WIBERG, K. \& TYSKLIND, M. Using soil function evaluation in multi-criteria decision analysis for sustainability appraisal of remediation alternatives. Science of The Total Environment, v 485-486, n.1, p. 785-791, 2014. http://dx.doi.org/10.1016/j.scitotenv.2014.01.087

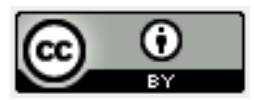

Artigo recebido em 05/12/2014 e aceito para publicação em 16/01/2015 DOI: http://dx.doi.org/ 10.14488/1676-1901.v15i3.1937 